gical problems may be nearing a practicable solution (Dr P. H. A. Sneath, University of Leicester). So far stress has been laid on digital computation and the problem of defining suitable parameters in fossil materials (Dr J. S. Hampton, University of Lancaster), but Dr D. Raup's (University of Rochester) eloquent cinefilm of the analogue computation of simulated shell form seemed to present, however, a previously overlooked and crucial intermediary step between classical taxonomy and digital programming as exemplified by Dr J. L. Cutbill (Cambridge). Hence the ASCOP computer facilities established in 1961 by the Institute of Geological Sciences may well find themselves in demand by palaeontologists in the future.

Dr G. E. Farrow (University of Hull) showed that comparative studies of the Recent bivalve Ceratoderma (Cardium) edule and ancient analogues are beginning to rationalize some long standing palaeoenvironmental arguments on the meaning of variations in shell sizes and structures. This work illustrates the biological deficiencies of many previous palaeontological reconstructions. Once again technological and molecular advances have overtaken biology before the palaeontologists' needs were fulfilled.

Isotopic studies were reviewed by $\mathrm{Dr}$ J. D. Hudson (University of Leicester) and exemplified in the Post-Pleistocene peat studies by Dr A. G. Smith (Queen's University, Belfast) and the Wealden palacoenvironmental analyses of Professor P. Allen (University of Reading). Though promising, this field is not without difficulties, particularly economic ones.

In summation, although technological advances have made a significant contribution to palaeontological studies, they have, until recently, so outstripped palaeontological thought that many confusing and ill conceived papers resulted. These brought ridicule to the implementation of the techniques and thereby hampered palaeontological advances. This meeting showed, however, that a new era of thoughtful experimentation and expression accompanied by a renewed vigour is firmly established.

\section{SURFACE CHEMISTRY}

\section{Sorption Phenomena}

from a Correspondent

THE interdisciplinary nature of the study of gas/solid interfaces was clear when members of the Vacuum. Thin Films and Surfaces Groups of the Institute of Physics and the Physical Society met at the University of Liverpool on January 6 . For many years, surface chemists have interpreted a wide variety of experimental data on chemisorption using a picture which describes chemisorption as the forma- tion of a local molecular structure, involving the adsorbate, and a few substrate atoms, very similar in essence to an ordinary molecule. Dr T. B. Grimley (University of Liverpool) explained how this picture of a surface molecule is contained in a general quantum theory of chemisorption, and stressed the importance of experimental methods designed to investigate the energy levels of electrons in such surface molecules. To illustrate the theory, Grimley presented numerical results for sodium, barium and sulphur atoms on the (100) faces of tungsten and nickel.

One experimental technique for investigating the energy levels of electrons in ordinary molecules is photoelectron spectroscopy. A monoenergetic photon source (the helium resonance line, or the $\mathrm{Mg} \mathrm{K} \alpha$ X-radiation for example) ejects an electron from the target molecule with kinetic energy equal to the difference between the photon source energy, and the binding energy of the electron in the molecule, so that a measurement of the kinetic energy of the ejected electron yields the binding energy. Some of the results obtained were reviewed by $\mathrm{Dr} \mathrm{M}$. Barber (AEI Scientific Apparatus I.td, Urmston), but the application of this technique to the study of adsorbed species is only just beginning. Its importance is that it gives direct information on key theoretical quantities.

Two other experimental techniques were discussed. Professor J. H. Leck (University of Liverpool) presented some results obtained with electron bombardment desorption (EBD), and Dr J. C. Riviere (AERE, Harwell) described some recent developments in Auger spectroscopy. In EBD, a monoenergetic electron beam (15-200 volts) ejects electrons from adorbed molecules, and positive ions, for example $\mathrm{O}^{-}$from adsorbed carbon monoxide, which desorb, can be studied. The situation is qualitatively similar to electron-molecule collisions in the gas phase, and the method yiclds information on the structure and bonding of surface molecules. Tightly bound species are, however, inactive in EBD; in the tungsten/ oxygen system for example. $\mathrm{O}^{+}$is not desorbed at low oxygen coverages. No such limitation exists with Auger spectroscopy, but here Rivière reported the unwelcome observation that the primary electron beam can interfere with the adsorption process; it causes enhanced adsorption of $\mathrm{CO}$ on silicon substrates. This is a reminder that sorption phenomena are likely to be just as complicated in 1971 as ever before. and the point was emphasized when $\mathrm{Mr}$ R. G. Forbes (University of Cambridge) ex-

\title{
Drugs and Brain Tryptophan
}

MANY drugs are known to increase the rate of synthesis of the neurotransmitter serotonin in the brain. These include (+)-amphetamine, reserpine, lithium salts in large quantities and intraventricular injections of dibutyryl cyclic AMP; serotonin synthesis is also accelerated in the brains of animals exposed to high environmental temperatures. Conversely. the administration of para-chlorophenylalanine inhibits the synthesis of brain serotonin. It has generally been assumed that such effects are mediated directly or indirectly through some influence on the enzyme tryptophan hydroxylase which catalyses the rate-limiting step in serotonin biosynthesis, the conversion of tryptophan to 5-hydroxytryptophan. The report by Tagliamonte et al. in next Wednesday's Nature New Biology, however, suggests an alternative and novel mechanism by which drugs may influence serotonin synthesis. These authors report that the administration of $(+)$-amphetamine, reserpine, lithium salts or dibutyryl cyclic AMP all caused significant increases in the concentration of tryptophan in rat brain. Exposure of animals to high environmental temperature $\left(40^{\circ} \mathrm{C}\right)$ also led to a large increase in brain tryptophan concentration. These increases ranged from a 60 per cent rise with reserpine to 300 per cent in rats exposed to $40^{\circ} \mathrm{C}$.
The increases in brain tryptophan did not simply reflect changes in the concentration of the amino-acid in plasma, because reserpine and dibutyryl cyclic AMP caused increases in brain tryptophan without any significant increase in plasma tryptophan. Exposure of animals to cold stress, or the administration of a variety of other psychotropic drugs, failed to influence either brain or plasma tryptophan concentrations. On the other hand, the administration of para-chlorophenylalanine led to decreased concentrations of brain and plasma tryptophan.

Because the concentrations of tryptophan in mammalian brain are below the Michaelis constant of tryptophan hydroxylase, the rate of serotonin synthesis in the brain may be very dependent on the availability of the amino-acid. It should be remembered, however, that the concentration of tryptophan in the whole brain may not necessarily reflect accurately the intracellular concentration of this amino-acid in serotonin containing neurones. Nevertheless, the results of Tagliamonte et al. suggest that changes in brain tryptophan may well be important factors in controlling the synthesis of serotonin. The question of how drugs and high environmental temperatures cause changes in brain tryptophan remains unsolved. 